\title{
Von Neumann Entropy Spectra and Entangled Excitations in Spin-Orbital Models
}

\author{
Wen-Long You, ${ }^{1,2}$ Andrzej M. Oleś, ${ }^{1,3}$ and Peter Horsch ${ }^{1}$ \\ ${ }^{1}$ Max-Planck-Institut für Festkörperforschung, Heisenbergstrasse 1, D-70569 Stuttgart, Germany \\ ${ }^{2}$ School of Physical Science and Technology, Soochow University, Suzhou, Jiangsu 215006, People's Republic of China \\ ${ }^{3}$ Marian Smoluchowski Institute of Physics, Jagellonian University, Reymonta 4, PL-30059 Kraków, Poland
}

(Dated: October 21, 2018)

\begin{abstract}
We consider the low-energy excitations of one-dimensional spin-orbital models which consist of spin waves, orbital waves, and joint spin-orbital excitations. Among the latter we identify strongly entangled spin-orbital bound states which appear as peaks in the von Neumann entropy (vNE) spectral function introduced in this work. The strong entanglement of bound states is manifested by a universal logarithmic scaling of the vNE with system size, while the vNE of other spin-orbital excitations saturates. We suggest that spin-orbital entanglement can be experimentally explored by the measurement of the dynamical spin-orbital correlations using resonant inelastic x-ray scattering, where strong spin-orbit coupling associated with the core hole plays a role.
\end{abstract}

PACS numbers: 75.10.Jm, 03.65.Ud, 03.67.Mn, 75.25.Dk

Introduction.-The spin-orbital interplay is one of the important topics in the theory of strongly correlated electrons [1]. In many cases, the intertwined spin-orbital interaction is decoupled by mean-field approximation, and the spin and orbital dynamics are independent from each other. Thus a spinonly Heisenberg model can be derived by averaging over the orbital state, which successfully explains magnetism and optical excitations in some materials, for instance in $\mathrm{LaMnO}_{3}$ [2]. But in others, especially in $t_{2 g}$ systems [3], the orbital degeneracy plays an indispensable role in understanding the lowenergy properties in the Mott insulators of transition metal oxides (TMOs), such as $\mathrm{LaTiO}_{3}$ [4], $\mathrm{LaVO}_{3}$ and $\mathrm{YVO}_{3}$ [5], and also in recently discussed $\mathrm{RbO}_{2}$ [6]. The well known cases are also strong spin-orbit coupling which leads to locally entangled states [7], and entanglement on the superexchange bonds in $\mathrm{K}_{3} \mathrm{Cu}_{2} \mathrm{~F}_{7}[8]$. For such models, the mean-field-type approximation and the decoupling of composite spin-orbital correlations fail and generate uncontrolled errors, even when the orbitals are polarized [9]. The strong spin-orbital fluctuations on the exchange bonds will induce the violation of the Goodenough-Kanamori rules [10]. Furthermore, the flavors may form exotic composite spin-orbital excitations.

Model and system. - A paradigmatic model derived for a TMO in Mott-insulating limit is the one-dimensional (1D) spin-orbital Hamiltonian, which reads

$$
H=-J \sum_{\mathbf{i}}\left(\vec{S}_{\mathbf{i}} \cdot \vec{S}_{\mathbf{i}+1}+x\right)\left(\vec{T}_{\mathbf{i}} \cdot \vec{T}_{\mathbf{i}+1}+y\right),
$$

where $\vec{S}_{\mathbf{i}}$ and $\vec{T}_{\mathbf{i}}$ are spin-1/2 and pseudospin-1/2 operators representing the spin and orbital degrees of freedom located at site $\mathbf{i}$, respectively, and we set below $J=1$. It is proposed that ultracold fermions in zig-zag optical lattices can reproduce an effective spin-orbital model [11]. For general $\{x, y\}$, the model (1) has an $\mathrm{SU}(2) \otimes S U(2)$ symmetry. An additional $\mathbf{Z}_{2}$ bisymmetry occurs by interchanging spin and orbital operators when $x=y$. In the case of $x=y=\frac{1}{4}$, Hamiltonian (11) reduces to a SU(4) symmetric model, which is exactly soluble by the Bethe ansatz [12, 13]. There are three Goldstone modes corresponding to separate spin and orbital excitation, as well as composite spin-orbital excitations in case of $J<0$, in contrast to a quadratic dependence of the energy upon the momentum in the long-wave limit for $J>0$. The spectra of elementary excitations are commonly not analytically soluble away from the SU(4) point. We will, however, show that the low-energy excitations can be analytically obtained in some specific phases in the case when $J>0$, and this offers a platform to study the spin-orbital entanglement. In this Letter, we go beyond the ideas developed for spin systems [14]. We demonstrate that spin-orbital entanglement entropy clearly distinguishes weakly correlated spin-orbital excitations from bound states and resonances by its magnitude and distinct scaling behavior. We propose how to connect the entanglement entropy with experimentally observable quantities of recently developed spectroscopies.

von Neumann entropy. - Currently, concepts from quantum information theory are being studied with the aim to explore many-body theory from another perspective and vice versa. A particularly fruitful direction is using quantum entanglement to shed light on exotic quantum phases [15, 16]. Entanglement entropy even distinguishes phases in the absence of conventional order parameters [17]. In general a manybody quantum system is subdivided into $A$ and $B$ parts and the entanglement entropy is the von Neumann entropy (vNE), $\mathcal{S}_{\mathrm{vN}}=-\operatorname{Tr}\left\{\rho_{A} \log _{2} \rho_{A}\right\}$, where $\rho_{A}=\operatorname{Tr}_{B}\{\rho\}$ is the reduced density matrix of the subspace $A$ and $\rho$ is the full density matrix. The vNE is bounded, $\mathcal{S}_{\mathrm{vN}} \leq \log _{2} \operatorname{dim} \rho_{A}$, and easy to calculate. Experimental determination appears harder, yet there are proposals involving transport measurements in quantum point contacts [18].

Interestingly the vNE scales proportionally to the boundary of the subregion obtained by the spatial partitioning [19]. The dependence of the boundary or area law can be traced back to study of black hole physics [20] and was extensively exploited for 1D spin chains [21]. If the block $A$ is of length $l$ in a system of length $L$ with periodic boundary condition, the vNE of gapped ground states is bounded as $\mathcal{S}_{l}=\mathcal{O}(1)$, while a logarithmic scaling $\mathcal{S}_{l}=c \log _{2} l+\mathcal{O}(1)(L \gg l \gg 1)$ has been proven to be universal property of the gapless phases in criti- 


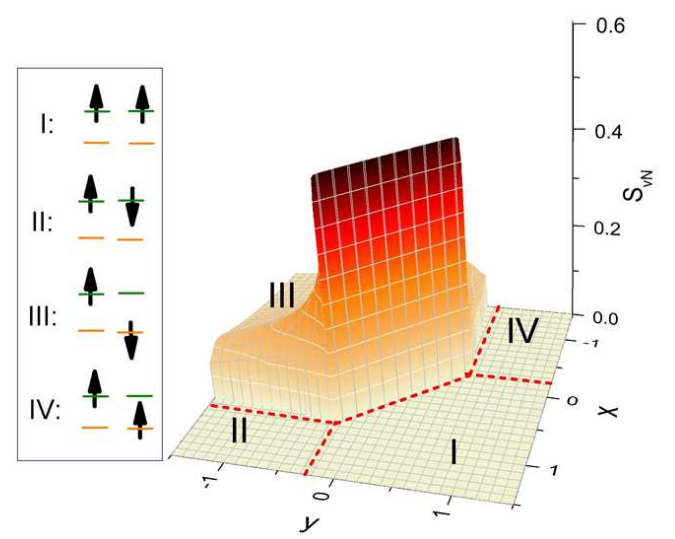

FIG. 1: (color online). Spin-orbital entanglement $\mathcal{S}_{\mathrm{vN}}$ in the groundstate of the spin-orbital model (1) as a function of $x$ and $y$ and system size $L=8$. The (red) dashed lines mark the critical lines determined by the fidelity susceptibility (see text). The two-site configurations in phases I-IV are shown on the left. The two orbitals per site are degenerate (their splitting is only for clarity of presentation).

cal systems by the underlying conformal field theory [22]. A violation of the area law is expected for the low-lying excited states of critical chains [23]. To date, measurements of the vNE for subdivision of degrees of freedom other than in spatial segmentation have not been fully explored. In a composite system containing spin and orbital operators, the decomposition of different flavors retains the real-space symmetries.

Phase diagram.- A quantum phase transition (QPT) is identified as a point of nonanalyticity of the ground state and associated expectation values in the thermodynamic limit. To shed light on the phase boundaries, we first consider two sites [24], $H_{12}=-\frac{1}{4}\left(\vec{S}_{12}^{2}-\vec{S}_{1}^{2}-\vec{S}_{2}^{2}+2 x\right)\left(T_{12}^{2}-\vec{T}_{1}^{2}-\vec{T}_{2}^{2}+2 y\right)$, where $\vec{S}_{12}=\vec{S}_{1}+\vec{S}_{2}$ and $\vec{T}_{12}=\vec{T}_{1}+\vec{T}_{2}$. A pair of spins (orbitals) can form either a singlet with $S_{12}=0\left(T_{12}=0\right)$ or a triplet with $S_{12}=1\left(T_{12}=1\right)$, and various combinations of quantum numbers correspond to different phases shown in Fig. 1 In phase I, the state with $S_{12}=1=T_{12}$ has the lowest energy, and thus the energy per bond is $e_{B}^{I} \geq e_{x y}=$ $-(x+1 / 4)(y+1 / 4)$. For a larger system with $L$ bonds, we have $E_{0}^{I}(H) \geq L e_{x y}$. On the other hand, taking a ferro-ferro state $|0\rangle$ as a variational state, $E_{0}^{I}(H) \leq L e_{x y}$. Therefore, the energy of phase I is exactly $E_{0}^{I}(H)=L e_{x y}$ and the ferroferro state is the corresponding ground state.

Without prior knowledge of order parameter, various characterizations from the perspective of quantum information theory can be used to identify phase boundaries. One often used tool is the vNE [25]. Tracing orbital degrees of freedom, we obtained the spin-orbital vNE $\mathcal{S}_{\mathrm{vN}}$ for the ground state of $L=8$ chain in the Hilbert subspace of $S_{z}=T_{z}=0$ [25]. However, here we find that the vNE of the ground state does not distinguish phase I from phase II or IV - all three phases having $\mathcal{S}_{\mathrm{vN}}=0$ (see Fig. 1). Therefore we use the quantum fidelity to quantify the phase diagram [26]. The fidelity defined
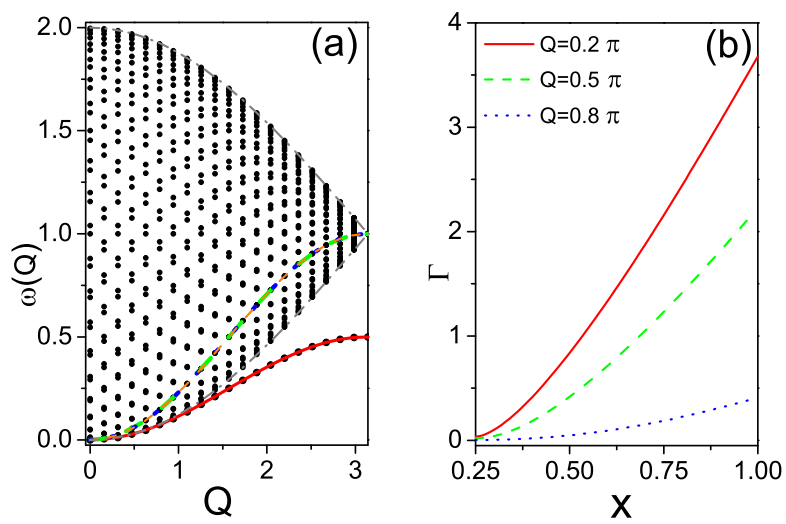

FIG. 2: (color online). (a) Energy spectra of 40-site spin-orbital system at $x=y=1 / 4$. Dashed lines in the spin-orbital continuum denote the spin, orbital and OBS excitation, all degenerate; the (red) solid line below corresponds to the BS. (b) The decay rate $\Gamma$ of the OBS for different momenta $Q$ with $y=x$ at $L \rightarrow \infty$.

as follows, $\mathcal{F}(\lambda, \delta \lambda)=\left|\left\langle\Psi_{0}(\lambda) \mid \Psi_{0}(\lambda+\delta \lambda)\right\rangle\right|$, is taken along a certain path $\{x(\lambda), y(\lambda)\}$ and reveals all phase boundaries. The fidelity susceptibility, $\chi_{\mathrm{F}} \equiv-(2 \ln \mathcal{F}) /\left.(\delta \lambda)^{2}\right|_{\delta \lambda \rightarrow 0}$, exhibits a peak at the critical point, and can be treated as a versatile order parameter in distinguishing ground states [27]. It signals the phase boundaries shown in Fig. 11 Remarkably, the phase diagram found from the fidelity susceptibility for larger systems is the same as the one for $L=2$.

Excitations. - In phase I of Fig. 1, with boundaries given by: $x+y=\frac{1}{2}, x=-\frac{1}{4}$ and $y=-\frac{1}{4}$, the spins and orbitals are fully polarized, and the ferro-ferro ground state $|0\rangle$ is disentangled, i.e., can be factorized into spin and orbital sector. It is now interesting to ask whether: (i) the vanishing spinorbital entanglement in the ground state implies a suppression of joint spin-orbital quantum fluctuations, and (ii) collective spin-orbital excitations can form. Using equation of motion method one finds spin (magnon) excitations with dispersion $\omega_{s}(q)=\left(\frac{1}{4}+y\right)(1-\cos q)$, and orbital (orbiton) excitations, $\omega_{t}(q)=\left(\frac{1}{4}+x\right)(1-\cos q)$ [28]. The stability of the orbitons (magnons) implies that $x>-\frac{1}{4}\left(y>-\frac{1}{4}\right)$, and determines the QPT between phases I and II (IV), respectively, while the spin-orbital coupling only renormalizes the spectra.

For our purpose, it is straightforward to consider the propagation of a pair of magnon and orbiton along the ferro-ferro chain, by simultaneously exciting a single spin and a single orbital. The translation symmetry imposes that total momentum $Q=2 m \pi / L(m=0, \cdots, L-1)$ is conserved during scattering. The scattering of magnon and orbiton with initial (final) momenta $\left\{\frac{Q}{2}-q, \frac{Q}{2}+q\right\}\left(\left\{\frac{Q}{2}-q^{\prime}, \frac{Q}{2}+q^{\prime}\right\}\right)$ and total momentum $Q$ is represented by the Green's function [29],

$$
G(Q, \omega)=\frac{1}{L} \sum_{q, q^{\prime}}\left\langle\left\langle S_{\frac{Q}{2}-q^{\prime}}^{+} T_{\frac{Q}{2}+q^{\prime}}^{+} \mid S_{\frac{Q}{2}-q}^{-} T_{\frac{Q}{2}+q}^{-}\right\rangle\right\rangle,
$$

for a combined spin $\left(S_{\frac{Q}{2}-q}^{-}\right)$and orbital $\left(T_{\frac{Q}{2}+q}^{-}\right)$excitation. 


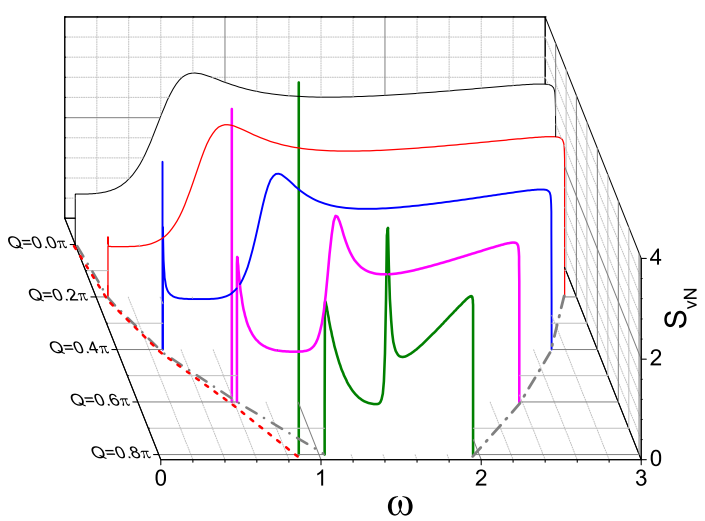

FIG. 3: (color online). The vNE distribution of 400-site spin-orbital system in subspace $P_{\mathrm{ST}}=1$ for $x=y=1 / 2$ and different momenta $Q$. Isolated vertical lines indicate the $\mathrm{BS}$, with dispersion given by the (red) dashed line. The OBS in the center of spectra is damped.

The spin-orbital continuum is given by $\Omega(Q, q)=\omega_{s}\left(\frac{Q}{2}-\right.$ $q)+\omega_{t}\left(\frac{Q}{2}+q\right)$. In the noninteracting case, the Green's function exhibits square-root singularities at the edges of the continuum [30]. Due to residual, attractive interactions spinorbital bound states (BSs) are shifted outside the continuum [24, 31, 32], see Fig. 2]a). The collective mode is determined by $1+\frac{1}{2 \pi} \int_{-\pi}^{\pi} d q\left(\cos \frac{Q}{2}-\cos q\right)^{2} /[\omega-\Omega(Q, q)]=0$. The analytic solution of this equation is tedious but straightforward. The collective BS with dispersion $\omega_{\mathrm{BS}}(Q)$ is well separated from the spinon-orbiton continuum [Fig. 2(a)] at large $Q$. In the long-wave limit the BS energy coincides with the Arovas-Auerbach line [33], i.e., the boundary of the continuum, yet the BS remains undamped for $x+y>\frac{1}{2}$.

In addition, a collective mode of spin-orbital resonances,

$$
|\Psi(Q)\rangle=\frac{1}{\sqrt{L}} \sum_{m, l} a_{l}(Q) e^{i Q m} S_{m}^{-} T_{m+l}^{-}|0\rangle,
$$

occurs inside the continuum. Here $0 \leq l \leq L-1$ denotes the distance between spin and orbital flips. Remarkably, the spin and orbital flips are glued together at the same site with $a_{l}(Q)=\delta_{l, 0}$ at the SU(4) point [28]. This coupled on-site BS (OBS) is a coherent superposition of local modes, all of them with equal weight. It has dispersion $\omega_{\mathrm{OBS}}(Q)=x+y-\frac{1}{2} \cos Q$, which is degenerate with both $\omega_{s}(Q)$ and $\omega_{t}(Q)$ at $x=y=\frac{1}{4}$, see Fig. 22 a). This is reminiscent of the degeneracy of the three Goldstone modes at the $\mathrm{SU}(4)$ point for $J=-1$ [12, 13]. Moving away from the SU(4) point, the OBS decays due to residual interactions into magnon-orbiton pairs, and the mean separation $\xi$ of spin and orbital excitations increases, i.e., $a_{l}(Q) \sim \exp (-l / \xi)$, leading in the thermodynamic limit to a finite linewidth defined by $\Gamma=\operatorname{Im} G^{-1}(Q, \omega)[34$. The decay rate of the spin-orbital OBS increases with growing $x>\frac{1}{4}$ and also for decreasing momenta $Q$, as seen in Fig. 2 (b).

Entropy spectral function.- To investigate the degree of entanglement of excited states, we introduce the vNE spectral
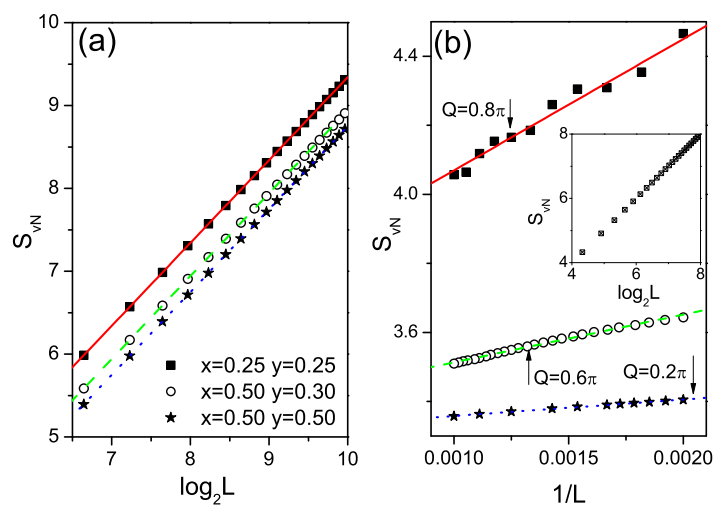

FIG. 4: (color online). (a) Scaling behavior of entanglement entropy $\mathcal{S}_{\mathrm{vN}}$ of the spin-orbital BSs for $Q=0.8 \pi$. Lines represent logarithmic fits $\mathcal{S}_{\mathrm{vN}}=\log _{2} L+c_{0}$, with $c_{0}=-0.659,-1.059,-1.251$, respectively. (b) The scaling behavior of entanglement entropy of the OBS for $x=y=1 / 2$. Lines are fitted by $\mathcal{S}_{\mathrm{vN}}=c_{1} / L+c_{0}$, with $c_{0}\left(c_{1}\right)=3.69$ (380.5), 3.37 (138.4) and 3.31 (47.6) for $Q=0.8 \pi$, $0.6 \pi$ and $0.2 \pi$. The inset shows the logarithmic behavior of $\mathcal{S}_{\mathrm{vN}}$ for the OBS with $Q=0.8 \pi$ and $x=y=1 / 4$.

function in the Lehmann representation,

$$
S_{\mathrm{vN}}(Q, \omega)=-\sum_{n} \operatorname{Tr}\left\{\rho_{s}^{(\mu)} \log _{2} \rho_{s}^{(\mu)}\right\} \delta\left\{\omega-\omega_{n}(Q)\right\},
$$

where $(\mu)=\left(Q, \omega_{n}\right)$ denote momentum and excitation energy, and $\rho_{s}^{(\mu)}=\operatorname{Tr}_{o}\left|\Psi_{n}(Q)\right\rangle\left\langle\Psi_{n}(Q)\right|$ is obtained by tracing the orbital degrees of freedom. Let us first consider the symmetric case, i.e., $x=y$. The Hilbert space can be divided into two subspaces characterized by the parity $P_{\mathrm{ST}}$ of the interchange of $S \leftrightarrow T$, which is odd or even. Translation symmetry allows us to express the reduced density matrix $\rho_{s}$ in a block-diagonal form, where each block corresponds to an irreducible representation labeled by total momentum $Q$ and parity of exchange symmetry $P_{\mathrm{ST}}$. The vNE can be obtained by diagonalizing separately these blocks. In particular, the nondegenerate eigenstates with odd parity can be explicitly cast in the form $\frac{1}{\sqrt{2}}\left(S_{Q / 2-q}^{-} T_{Q / 2+q}^{-}-S_{Q / 2+q}^{-} T_{Q / 2-q}^{-}\right)|0\rangle$. Consequently, the singlet-like pair results in $\mathcal{S}_{\mathrm{vN}}=1$. For other spin-orbital eigenstates with $P_{\mathrm{ST}}=1, \mathcal{S}_{L} \geq 1$, except the pure spin and orbital waves. Interestingly, we find that the parity is still conserved in subspace $Q=0$ for $x \neq y$. The strongly entangled spin-orbital BSs are reflected by peaks in the von Neumann spectra $S_{\mathrm{vN}}(\omega)$, shown in Fig. 3. As momentum $Q$ decreases, the OBS-peak in the center of spectra gets broader, implying a shorter lifetime.

Inspection of vNE spectra shows that the entanglement reaches a local maximum at the BSs. Finite size scaling of vNE of spin-orbital BSs reveals the asymptotic logarithmic scaling $\mathcal{S}_{\mathrm{vN}}=\log _{2} L+c_{0}$ shown in Fig. 4(a). The same logarithmic scaling is found for the OBS at the SU(4) point $x=y=\frac{1}{4}$, as seen in the inset of Fig. 4(b). However, far away from the SU(4) point the scaling is entirely 


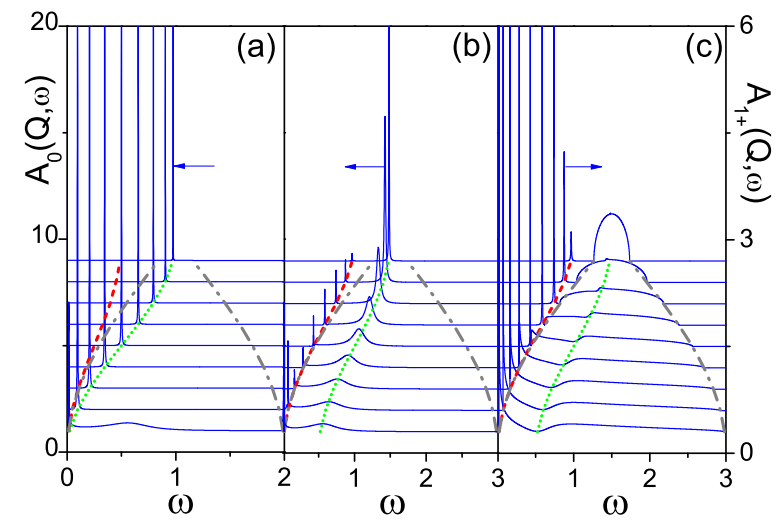

FIG. 5: (color online). The spectral function of the on-site excitation $A_{0}(Q, \omega)$ for: (a) $x=y=1 / 4$, (b) $x=y=1 / 2$; (c) the nearestneighbor $A_{1+}(Q, \omega)$ for $x=y=1 / 2$. The momenta range from $\pi / 10$ (bottom) to $9 \pi / 10$ (top); the peak broadening is $\eta=0.01$. Dashed (red) and dotted (green) lines correspond to the BS and OBS, while gray dash-dot lines indicate the boundaries of the continuum.

different and the entropy of the OBS scales as a power law, $\mathcal{S}_{\mathrm{vN}}=c_{1} / L+c_{0}$, as seen in Fig. 4(b). This change of scaling from logarithmic to power law in $1 / L$ is controlled by the correlation length $\xi$ measuring the average distance of spin and orbital excitations in the OBS wave function (3). From Eq. (3) and $a_{l}(Q) \sim \exp (-l / \xi)$ we obtain,

$$
S_{\mathrm{vN}} \simeq \log _{2}\{L /(1+\xi)\},
$$

which yields $\log _{2} L$ at $x=y=1 / 4$ where $\xi=0$. As $\xi$ increases the correction to the vNE is $\propto-\log _{2}(1+\xi)$. Far away from the $\mathrm{SU}(4)$ point, the OBS is damped and $\xi$ becomes extensive, i.e., $\xi / L \approx \tilde{c_{0}}-\tilde{c_{1}} / L$, and the vNE approaches a finite value with a correction $\propto 1 / L$ as shown in Fig. 4(b). This close correspondence of the vNE of bound states and the correlation length $\xi$ suggests to use the dynamic spin-orbital correlation function as a probe of spin-orbital entanglement and as a qualitative measure of the vNE spectra.

Spectral functions.- Returning to TMOs, one realizes that joint spin-orbital excitations are not created in the ferro-ferro ground state in photoemission spectroscopy because of spinconservation. On the contrary, resonant inelastic x-ray scattering (RIXS) [35-39] is in principle able to measure the spectral function of the coupled spin-orbital excitations at distance $l$,

$$
A_{l}(Q, \omega)=\frac{1}{\pi} \lim _{\eta \rightarrow 0} \operatorname{Im}\left\langle 0\left|\Gamma_{Q}^{(l) \dagger} \frac{1}{\omega+E_{0}-H-i \eta} \Gamma_{Q}^{(l)}\right| 0\right\rangle .
$$

Here $\Gamma_{Q}^{(0)}=\frac{1}{\sqrt{L}} \sum_{j} e^{i Q j} S_{j}^{-} T_{j}^{-}$is the local excitation operator for an on-site spin-orbital excitation. We use as well $\Gamma_{Q}^{\left(1_{ \pm}\right)}=\frac{1}{\sqrt{2 L}} \sum_{j} e^{i Q j}\left(S_{j+1}^{-} \pm S_{j-1}^{-}\right) T_{j}^{-}$for the nearestneighbor excitation. In the RIXS process an electron with spin up is excited by the incoming x-rays from a deep-lying core level into the valence shell. For the time of its existence the core hole generates a Coulomb potential and a strong spinorbit coupling that allows for the non-conservation of spin. Next the hole is filled by an electron from the occupied valence band under the emission of an X-ray. This RIXS process creates a joint spin-valence excitation with momentum $Q_{\text {in }}-Q_{\text {out }}$ and energy $\omega_{\text {in }}-\omega_{\text {out }}$, which can unveil the spectral function of the spin-orbital excitation.

The on-site spectral function $A_{0}(Q, \omega)$ shown in Figs. 5 (a,b) highlights the OBS. At the SU(4) point [Fig. 5(a)] it appears as a $\delta$-function, $A_{0}(Q, \omega)=\delta\left\{\omega-\omega_{\mathrm{OBS}}(Q)\right\}$, whereas in Fig. 5 b) the OBS is damped and its intensity decreases strongly with $Q$. In the latter figure the BS at the low energy side of the continuum appears as weak additional feature, while it is absent in (a), i.e., at the $\mathrm{SU}(4)$ point. The nearest neighbor spectral function $A_{1+}(Q, \omega)$ in Fig. 5] c) shows both the spin-orbital continuum and the BS outside of the continuum. Notably, comparing with the vNE spectral function in Fig. 3, we find the same characteristic energies and similar intensity features as in the RIXS spectra. The spectral function provides information of various correlations, which are ingredients to derive the reduced density matrices [40].

Summary. - In this Letter, we study a spin-orbital system and extend the analysis of entanglement to excited states by introducing the vNE spectral function. Our study demonstrates that even in cases where the ground state of a spinorbital chain is fully disentangled, e.g., in the ferro-ferro state, (i) the spin-orbital excitations are in general entangled, (ii) maximal spin-orbital entanglement occurs for BSs which appear as sharp peaks in the vNE spectra, and (iii) the vNE of undamped BSs exhibits a logarithmic dependence on the chain length $L$. We propose to study the dynamic spin-orbital correlation function as a qualitative measure of the vNE spectra, and suggest to use here RIXS as a promising technique.

W-L.Y. acknowledges support by the National Natural Science Foundation of China (NSFC) under Grant No. 11004144. A.M.O. acknowledges support by the Polish National Science Center (NCN) under Project No. N202 069639.

[1] Y. Tokura and N. Nagaosa, Science 288, 462 (2000).

[2] L.F. Feiner and A.M. Oleś, Phys. Rev. B 59, 3295 (1999); N.N. Kovaleva, A.M. Oleś, A.M. Balbashov, A. Maljuk, D.N. Argyriou, G. Khaliullin, and B. Keimer, ibid. 81, 235130 (2010).

[3] Sergey Krivenko, Phys. Rev. B 85, 064406 (2012).

[4] G. Khaliullin and S. Maekawa, Phys. Rev. Lett. 85, 3950 (2000).

[5] Peter Horsch, Andrzej M. Oleś, Louis Felix Feiner, and Giniyat Khaliullin, Phys. Rev. Lett. 100, 167205 (2008).

[6] K. Wohlfeld, M. Daghofer, and A.M. Oleś, Europhys. Lett. 96, 27001 (2011).

[7] G. Jackeli and G. Khaliullin, Phys. Rev. Lett. 102, 017205 (2009).

[8] W. Brzezicki and A.M. Oleś, Phys. Rev. B 83, 214408 (2011).

[9] K. Wohlfeld, M. Daghofer, S. Nishimoto, G. Khaliullin, and J. van den Brink, Phys. Rev. Lett. 107, 147201 (2011). 
[10] Andrzej M. Oleś, Peter Horsch, Louis Felix Feiner, and Giniyat Khaliullin, Phys. Rev. Lett. 96, 147205 (2006).

[11] G. Sun, G. Jackeli, L. Santos, and T. Vekua, arXiv: 1112.5082 (unpublished).

[12] You-Quan Li, Michael Ma, Da-Ning Shi, and Fu-Chun Zhang, Phys. Rev. B 60, 12781 (1999).

[13] B. Sutherland, Phys. Rev. B 12, 3795 (1975).

[14] Vincenzo Alba, Maurizio Fagotti, and Pasquale Calabrese, J. Stat. Mech. 10, P10020 (2009).

[15] Hui Li and F.D.M. Haldane, Phys. Rev. Lett. 101, 010504 (2008).

[16] Hong Yao and Xiao-Liang Qi, Phys. Rev. Lett. 105, 080501 (2010).

[17] A. Kitaev and J. Preskill, Phys. Rev. Lett. 96, 110404 (2006).

[18] I. Klich and L. Levitov, Phys. Rev. Lett. 102, 100502 (2009).

[19] J. Eisert, M. Cramer and M.B. Plenio, Rev. Mod. Phys. 82, 277 (2010).

[20] Luca Bombelli, Rabinder K. Koul, Joohan Lee, and Rafael D. Sorkin, Phys. Rev. D 34, 373 (1986); Mark Srednicki, Phys. Rev. Lett. 71, 666 (1993); S.W. Hawking, J. Maldacena, A. Strominger, J. High Energy Phys. 05, 001 (2001).

[21] Luigi Amico, Rosario Fazio, Andreas Osterloh, and Vlatko Vedral, Rev. Mod. Phys. 80, 517 (2008).

[22] Christoph Holzhey, Finn Larsen, and Frank Wilczek, Nucl. Phys. B 424, 443 (1994).

[23] Lluís Masanes, Phys. Rev. A 80, 052104 (2009).

[24] J. van den Brink, W. Stekelenburg, D.I. Khomskii, G.A. Sawatzky, and K. I. Kugel, Phys. Rev. B 58, 10276 (1998).

[25] Yan Chen, Z.D. Wang, Y.Q. Li, and F.C. Zhang, Phys. Rev. B 75, 195113 (2007).
[26] Shi-Jian Gu, Int. J. Mod. Phys. B 24, 4371 (2010).

[27] Wen-Long You, Ying-Wai Li, and Shi-Jian Gu, Phys. Rev. E 76, 022101 (2007).

[28] Alexander Herzog, Peter Horsch, Andrzej M. Oleś, and Jesko Sirker, Phys. Rev. B 83, 245130 (2011).

[29] Michael Wortis, Phys. Rev. 132, 85 (1963).

[30] T. Schneider, Phys. Rev. B 24, 5327 (1981).

[31] Jan Bała, Andrzej M. Oleś, and George A. Sawatzky, Phys. Rev. B 63, 134410 (2001).

[32] S. Cojocaru and A. Ceulemans, Phys. Rev. B 67, 224413 (2003).

[33] D.P. Arovas and A. Auerbach, Phys. Rev. B 52, 10114 (1995).

[34] Dyson equation reads $G^{-1}(Q, \omega)=\omega-\omega_{\text {oBs }}(Q)-\Sigma(Q, \omega)$, with the total self-energy $\Sigma(Q, \omega)$. The real part $\operatorname{Re} G^{-1}(Q, \omega)$ identifies the effective energy pole, and the imaginary part of self-energy is the inverse of lifetime $\tau$, i.e., $\Gamma=\operatorname{Im} \Sigma(Q, \omega)$.

[35] S. Ishihara and S. Maekawa, Phys. Rev. B 62, 2338 (2000).

[36] M.W. Haverkort, Phys. Rev. Lett. 105, 167404 (2010).

[37] L.J.P. Ament, M. van Veenendaal, T.P. Devereaux, J.P. Hill, and J. van den Brink, Rev. Mod. Phys. 83, 705 (2011).

[38] Filomena Forte, Luuk J.P. Ament, and Jeroen van den Brink, Phys. Rev. Lett. 101, 106406 (2008).

[39] J. Schlappa, K. Wohlfeld, K.J. Zhou, M. Mourigal, M.W. Haverkort, V. N. Strocov, L. Hozoi, C. Monney, S. Nishimoto, S. Singh, A. Revcolevschi, J.-S. Caux, L. Patthey, H.M. Rønnow, J. van den Brink, and T. Schmitt, Nature 485, 82 (2012).

[40] I. Peschel and V. Eisler, J. Phys. A 42, 504003 (2009). 